TAIWANESE JOURNAL OF MATHEMATICS

Vol. 2, No. 3, pp. 347-352, September 1998

\title{
PSEUDOCONVEXITY OF RIEMANN DOMAINS OVER A PRODUCT OF COMPLEX PLANES
}

\author{
Kwang Ho Shon, Sang Keun Lee, and Ern Geun Kwon
}

\begin{abstract}
This paper is concerned with the pseudoconvex Riemann domain over the product space $\mathbb{C}^{\mathbb{N}}$. We investigate that the pseudoconvex Riemann domain is the product of $\mathbb{C}^{\mathbb{N}}-\{1,2, \cdots, n\}$ and a pseudoconvex Riemann domain over $\mathbb{C}^{n}$.
\end{abstract}

\section{INTRODUCTION}

Recenty, H. Kazama [8] proved that any Stein neighborhood of $\mathbb{C}^{k} \times \mathbb{R}^{l}(1 \leq$ $k, l<\infty)$ in $\mathbb{C}^{k} \times \mathbb{C}^{l}$ is a product of $\mathbb{C}^{k}$ and a domain of holomorphy in $\mathbb{C}^{l}$ and showed that $\mathbb{C}^{k} \times \mathbb{R}^{l}$ has no Stein neighborhood bases in $\mathbb{C}^{k} \times \mathbb{C}^{l}$. More recently, S. Ohgai and K. H. Shon [17] proved that a domain of holomorphy in the product space of the complex Glassmann manifold $M_{n, k}$ and $\mathbb{C}^{l}$ containing $M_{n, k} \times G\left(G \subset \mathbb{R}^{l}\right)$ is a product of $M_{n, k}$ and a Stein connected open neighborhood of $G$ in $\mathbb{C}^{l}$. J. Kajiwara, S. Ohgai and K. H. Shon [7] extended this result to complex Lie groups. M. Nishihara, K. H. Shon and N. Sugawara [14] solved this problem in a locally convex Hausdorff space over $\mathbb{C}$. H. Hamada and J. Kajiwara [2] solved this problem in the related pseudoconvex domain. Most recently, K. H. Shon [19] proved that a pseudoconvex Riemann domain containing the product space at a real domain and a complex vector space $E$ is the product space of a pseudoconvex Riemann domain and $E$. The present paper is aimed at a Schlicht Riemann domain over the Cartesian product $\mathbb{C}^{\mathbb{N}}$ of a countable number of complex planes.

Received December 26, 1996.

Communicated by S.-Y. Shaw.

1991 Mathematics Subject Classification: 32F15, 46E50.

Key words and phrases: Pseudoconvex domain, Riemann domain.

The first author was supported by Non Directed Research Fund, Korea Research Foundation, 1996 and the present studies were supported in part by the Basic Science Research lnstitute Program, Korean Ministry of Education, 1995, Project No. BSRI-95-1411 . 


\section{Preliminaries and Notations}

Let $(\Omega, \varphi)$ be a Riemann domain over $\Omega$, i.e., $\Omega$ is a Hausdorff topological space and an analytic map $\varphi: \Omega \rightarrow E$ is locally an homeomorphism. Let $A$ be a subset of $E$. A map $\mathcal{S}: A \rightarrow \Omega$ is called a section of $(\Omega, \varphi)$ over $A$ if $\mathcal{S}: A \rightarrow \Omega$ is a continuous mapping such that $\mathcal{S} \circ \varphi=i d_{A}$. Let $E$ be a locally convex Haudorff space over $\mathbb{C},(\Omega, \varphi)$ be a (connected) Riemann domain over $E$ and $\operatorname{cs}(E)$ be the set of all continuous seminorms on $E$. We define the (boundary) distance functions $d_{\Omega}^{\alpha}: \Omega \rightarrow[0,+\infty]$ for $\alpha \in \operatorname{cs}(E)$, and $\delta_{\Omega}: \Omega \times E \rightarrow(0,+\infty]$, as follows:

$$
\begin{gathered}
d_{\Omega}^{\alpha}(x)=\sup \left(\left\{r>0: \text { there is a section } \sigma: B_{E}^{\alpha}(\varphi(x), r) \rightarrow \Omega\right.\right. \\
\text { with } \sigma \circ \varphi(x)=x\} \cup\{0\}, \\
\delta_{\Omega}(x, a)=\sup \left\{r>0: \text { there is a section } \sigma: D_{E}(\varphi(x), a, r) \rightarrow \Omega\right. \\
\text { with } \sigma \circ \varphi(x)=x\},
\end{gathered}
$$

where for $\xi, a \in E$ and $r>0$ we write

$$
\begin{gathered}
B_{E}^{\alpha}(\xi, r)=\{\xi+b: b \in E, \alpha(b)<r\}, \\
D_{E}(\xi, a, r)=\{\xi+\lambda a: \lambda \in \mathbb{C},|\lambda|<r\} .
\end{gathered}
$$

We let $D(\zeta, r)$ be the disc with center $\zeta$ and radius $r, \mathcal{H}(\Omega)$ the complex holomophic mappings in $\Omega$. The domain $\Omega$ is said to be pseudoconvex if the function $-\log \delta_{\Omega}$ is plurisubharmonic on $\Omega \times E$ (see J. Mujica [11,12]). The following is a well-known result (cf. P. Noverraz [15] or M. Schottenloher [18]).

Proposition 2.1. Let $(\Omega, \varphi)$ be a Riemann domain over a locally convex space $E$. Then the following conditions are equivalent:

(1) $\Omega$ is a pseudoconvex domain.

(2) For any $\alpha \in c s(E),-\log d_{\Omega}^{\alpha}$ is plurisubharmonic on $\Omega$.

\section{Pluripolar Sets on Pseudoconvex Domains Over Locally Convex Spaces}

Let $E_{1}$ and $E_{2}$ be locally convex spaces over $\mathbb{C}$. We introduce the following definition. A set $P$ in $\mathbb{C}$ is polar if, on some (or every) open set $U \supset P$, there is a subharmonic $u$ on $U$ (which may be chosen $\leq 0$ if $U$ is bounded) such that $P \subset u^{-1}(-\infty)$. Polar subsets of an open set $\Omega$ in a locally convex space $E$ have several possible definitions (cf. M. Hervé [3, 4], P. Noverraz [16]). 
Definition 3.1. A subset $W$ of an open set $X$ in a locally convex space is pluripolar in $X$ if there is a plurisubharmonic function $p$ on $X$ (and $<0$ ) such that $W \subset p^{-1}(-\infty)$.

We have proved the following theorem.

Theorem 3.2([19]). Let $(\Omega, \varphi)$ be a Riemann domain over $E=E_{1} \times E_{2}$. Suppose that there exist an open set $G$ of $E_{2}$ and a section $\mathcal{S}: E_{1} \times G \rightarrow \Omega$. Then $\Omega$ is a pseudoconvex domain if and only if there exist a pseudoconvex Riemann domain $V$ over $E_{2}$ and a biholomorphic mapping $\sigma: E_{1} \times V \rightarrow \Omega$ such that $V=\varphi^{-1}\left(\{0\} \times E_{2}\right)$ and $\left(i d_{E_{1}},\left.\varphi\right|_{V}\right)=\varphi \circ \sigma$. Furthermore, if $\varphi$ is injective, then $\Omega=E_{1} \times V$.

Let $(\Omega, \varphi)$ be a Riemann domain over $\mathbb{C}^{\mathbb{N}}$ such that $\mathcal{H}(\Omega)$ separates the points of $\Omega$. M. Matos [10] proved that $(\Omega, \varphi)$ is a domain of holomorphy if and only if there exists a positive integer $n$ such that $(\Omega, \varphi)$ is of order $n$ in $\Omega$ and $\left(\Omega_{n}, \varphi_{n}\right)$ is a manifold of holomorphy spread over $\mathbb{C}^{n}$, where $\varphi_{n}=\left.\varphi\right|_{\Omega_{n}}$ and $\Omega_{n}=\varphi^{-1}\left[\pi_{n} \circ \varphi(\Omega)\right]$, and $\pi_{n}$ denotes the projection mapping from $\mathbb{C}^{\mathbb{N}}$ onto the space of the first $n$ variables. A. Hirschowitz[5] proved it for the case in which $(\Omega, \varphi)$ is an open subset of $\mathbb{C}^{\mathbb{N}}$.

Theorm 3.3. Let $(\Omega, \varphi)$ be a Schlicht Riemann domain over $\mathbb{C}^{\mathbb{N}}$. If $\Omega$ is a pseudoconvex domain, then there exists a number $n \in \mathbb{N}$ and a psedoconvex Riemann domain $\left(V,\left.\varphi\right|_{V}\right)$ over $\mathbb{C}^{n}$ such that $\Omega=\mathbb{C}^{\mathbb{N}-\{1,2, \cdots, n\}} \times V$.

Proof. For $x \in \Omega$, there is $\alpha \in c s\left(\mathbb{C}^{\mathbb{N}}\right)$ with $d_{\Omega}^{\alpha}(x) \geq 1$. Thus for $z=\left(z_{i}\right)$, there exist $n \in \mathbb{N}$ and $c>0$ such that

$$
c\left(\sup _{1 \leq i \leq n}\left|z_{i}\right|\right) \geq \alpha(z) .
$$

Hence there exists a section

$$
\mathcal{S}: B_{\mathbb{C}^{\mathbb{N}}}^{\alpha}(\varphi(x), 1) \rightarrow \Omega
$$

satisfying $\mathcal{S} \circ \varphi(x)=x$. In fact, we have

$$
\begin{aligned}
B_{\mathbb{C}^{\mathbb{N}}}^{\alpha}(\varphi(x), 1)= & \left\{\varphi(x)+\zeta \in \mathbb{C}^{\mathbb{N}}: \alpha(\zeta)<1\right\} \\
& \supset\left\{\varphi(x)+\zeta \in \mathbb{C}^{\mathbb{N}}: c\left(\sup _{1 \leq i \leq n}\left|\zeta_{i}\right|\right)<1\right\}
\end{aligned}
$$




$$
\begin{aligned}
= & \left\{\varphi(x)+\left(\zeta_{1}, \zeta_{2}, \cdots, \zeta_{n}, \zeta_{n+1}, \cdots\right) \in \mathbb{C}^{\mathbb{N}}:\left|\zeta_{i}\right|<\frac{1}{c},\right. \\
& \left.i=1,2, \cdots, n, \zeta_{j} \in \mathbb{C}, j=n+1, n+2, \cdots\right\} \\
= & \varphi(x)+\left\{\left(\zeta_{1}, \zeta_{2}, \cdots, \zeta_{n}, 0,0, \cdots\right) \in \mathbb{C}^{\mathbb{N}}:\left|\zeta_{i}\right|<\frac{1}{c}, i=1,2, \cdots, n\right\} \\
& +\left\{\left(0,0, \cdots, 0, \zeta_{n+1}, \zeta_{n+2}, \cdots\right) \in \mathbb{C}^{\mathbb{N}}: \zeta_{j} \in \mathbb{C}, j=n+1, n+2, \cdots\right\} \\
= & \left(p_{1} \circ \varphi(x), p_{2} \circ \varphi(x), \cdots, p_{n} \circ \varphi(x), 0,0, \cdots, 0, \cdots\right) \\
& +\left(0,0, \cdots, 0, p_{n+1} \circ \varphi(x), p_{n+2} \circ \varphi(x), \cdots\right) \\
& +\left\{\left(\zeta_{1}, \zeta_{2}, \cdots, \zeta_{n}, 0,0, \cdots, 0, \cdots\right) \in \mathbb{C}^{\mathbb{N}}:\left|\zeta_{i}\right|<\frac{1}{c}, i=1,2, \cdots, n\right\} \\
& +\left\{\left(0,0, \cdots, 0, \zeta_{n+1}, \zeta_{n+2}, \cdots\right) \in \mathbb{C}^{\mathbb{N}}: \zeta_{j} \in \mathbb{C}, j=n+1, n+2, \cdots\right\} \\
= & \mathbb{C}^{\mathbb{N}-\{1,2, \cdots, n\}} \times \prod_{j=1}^{n} D\left(p_{j} \circ \varphi(x), \frac{1}{c}\right) .
\end{aligned}
$$

That is,

$$
\left.\mathcal{S}\right|_{\mathbb{C}^{\mathbb{N}-\{1,2, \cdots, n\}} \times \prod_{j=1}^{n} D\left(p_{j} \circ \varphi(x), \frac{1}{c}\right)}: B_{\mathbb{C}^{\mathbb{N}}}^{\alpha}(\varphi(x), 1) \rightarrow \Omega
$$

is a section satisfying $\left.\mathcal{S}\right|_{\mathbb{C}^{\mathbb{N}-\{1,2, \cdots, n\}} \times \prod_{j=1}^{n} D\left(p_{j} \circ \varphi(x), \frac{1}{c}\right)} \circ \varphi(x)=x$. From Theorem 3.2 , we have the result.

Deflnition 3.4. Let $\Omega$ be an open set in a locally convex space $E$. A set $A \subset \Omega$ is unipolar in $\Omega$ if:

(1) for every complex line $L$ in $E$ and every connected component $U$ of $L \cap \Omega, A \cap U$ is either polar or $A \cap U=U$,

(2) $\AA=\emptyset$ or, equivalently, $A$ does not include any connected component of $\Omega$.

Lemma 3.5. $\mathbb{R}$ is not a polar set in $\mathbb{C}$.

Proof. Assume that $\mathbb{R}$ is a polar set in $\mathbb{C}$. Then $\mathbb{C}-\mathbb{R}$ is connected. This is a contradiction.

Let $E(\mathbb{R})$ be any locally convex Hausdorff space over $\mathbb{R}$. For any $(a, b),(c, d) \in$ $E(\mathbb{R}) \times E(\mathbb{R})$ and any complex number $\lambda=\alpha+\sqrt{-1} \beta$, we define

$$
\begin{gathered}
(a, b)+(c, d)=(a+c, b+d), \\
\lambda \cdot(a, b)=(\alpha a-\beta b, \beta a+\alpha b) .
\end{gathered}
$$


Then $E(\mathbb{R}) \times E(\mathbb{R})$ can be turned into a vector space over $\mathbb{C}$. If $\mathcal{B}$ is a fundamental system of 0-neighborhoods of $E(\mathbb{R})$ consisting of convex balanced sets, then the family

$$
\mathcal{B}(\mathbb{C})=\left\{\lambda(x, y) ; x \in V_{1}, y \in V_{2},|\lambda| \leq 1\right\}_{V_{1}, V_{2} \in \mathcal{B}}
$$

forms a fundamental system of 0-neighborhoods of $E(\mathbb{R}) \times E(\mathbb{R})$ consisting of convex balanced sets (see, J. E. Colombeau [1]). We denote by $E(\mathbb{C})$ the vector space $E(\mathbb{R}) \times E(\mathbb{R})$ equipped with the topology defined by $\mathcal{B}(\mathbb{C})$. Then $E(\mathbb{C})$ is also a locally convex Hausdorff space over $\mathbb{C}$, and $E(\mathbb{C})$ is called the complexification of $E(\mathbb{R})$.

Proposition 3.6. Let $E$ be a locally convex space over $\mathbb{R}, G$ be on open subset of $E$ and $E(\mathbb{C})$ be the complexification of $E$. If $\Omega$ is an open subset of $E(\mathbb{C})$ with $G \subset \Omega$, then $G$ is not a unipolar set in $\Omega$.

Proof. Assume that $G$ is unipolar in $\Omega$. Then there exists a complex line $L$ in $E$ with $L \cap G \neq \emptyset$. Since $G \cap L \subset E$, we have

$$
L \cap G=L \cap E \cap G \subset L \cap E \subset L \cap E(\mathbb{C}) .
$$

Since $L \cap G \neq \emptyset$ and $L \cap G \nsubseteq L \cap \Omega, L \cap G$ is a polar set in $L \cap \Omega$. This contradicts Lemma 3.5.

\section{REFERENCES}

1. J. E. Colombeau, Differential Calculus and Holomorphy, North-Holland Math. Studies 64, North-Holland Publ. Co., Amsterdam, 1982.

2. H. Hamada and J. Kajiwara, Ensembles totalement réels et domains pseudoconvexes, Mem. Fac. Sci. Kyushu Univ. Ser. A 39-2 (1985), 243-247.

3. M. Hervé, Analytic and Plurisubharmonic Functions in Finite and Infinite Dimensional Spaces, Lecture Notes in Math., no. 198, Springer-Verlag, Berlin, 1971.

4. M. Hervé, Analyticity in Infinite Dimensional Spaces, de Gruyter Studies in Mathematics 10, Walter de Gruyter, Berlin, 1989.

5. A. Hirschowitz, Remarques sur les ouverts d'holomorphie d'un produit dénombrable de droites, Ann. Inst. Fourier (Grenoble) 19-1 (1969), 219-229.

6. M. Jarnicki and P. Pflug, Invariant Distances and Metrics in Complex Analysis, Walter de Gruyter, Berlin, 1993.

7. J. Kajiwara, S. Ohgai and K. H. Shon, Groupes de Lie complexes et fonctions holomorphes. Mem. Fac. Sci. Kyushu Univ. Ser. A 39-1 (1985), 127-132. 
8. H. Kazama, Note on Stein neighborhoods of $\mathbb{C}^{k} \times \mathbb{R}^{l}$, Math. Rep. College Gen. Educ. Kyushu Univ. 14-1 (1983), 47-55.

9. P. Lelong, Séminaire de Math., Fonctionnelle Analytiques et Fonctions Entières (n variables), Les Presses de L'Université de Montréal, Canada.

10. M. Matos, The envelope of holomorphy of Riemann domains over a countable product of complex planes, Trans. Amer. Math. Soc. 167 (1972), 379-387.

11. J. Mujica, Holomorphic approximation in infinite-dimensional Riemann domains, Studia Math. 82 (1985), 107-134.

12. J. Mujica, Complex Analysis in Banach Spaces, North-Holland Mathematics Studies 120, North-Holland Pulb. Co., Amsterdam, 1986.

13. M, Nishihara, On the indicator of growth of entire functions of exponential type in infinite dimensional spaces and the Levi problem in infinite dimensional projective spaces, Portugal Math. J. 52 (1995), 61-94.

14. M. Nishihara, K. H. Shon and N. Sugawara, On pseudoconvex neighborhoods of real subspaces in some locally convex spaces over $\mathbb{C}$, Mem. Fac. Sci. Kyushu Univ. Ser. A 39-1 (1985), 85-93.

15. P. Noverraz, Pseudo-Convexité, Convexité Polynomiale et Domaines d'Holomorphie en Dimension Infinite, North-Holland Math. Studies 3, NorthHolland Publ. Co., New York, 1973.

16. ___ Fonctions plurisousharmoniques et analytiques dans les espaces vectoriels topologiques Complexes, Ann Inst. Fourier (Grenoble) 19-2 (1969), 419-493.

17. S. Ohgai and K. H. Shon, Stein neighborhoods of real axis, Mem. Fac. Sci. Kyushu Univ. Ser. A 38-2 (1984), 199-202.

18. M. Schottenloher, Das Levi problem in unendlichdimensionalen Räumen mit Schauder zerlegung, Habilitations Schrift, Universität, München, 1974.

19. K. H. Shon, On Riemann domains containing a certain real domain, Complex Variables Theory Appl. 31 (1996), 27-35.

Kwang Ho Shon

Department of Mathematics, College of Natural Sciences

Pusan National University, Pusan 609-735, Korea

Sang Keun Lee

Department of Mathematics Education, Gyengsang National University

Chinju 660-701, Korea

and

Ern Geun Kwon

Depatment of Mathematics Education, Andong National University

Andong 760-749, Korea 